Ergod. Th. \& Dynam. Sys. (1985), 5, 89-105

Printed in Great Britain

\title{
An invariant for bounded-to-one factor maps between transitive sofic subshifts
}

\author{
MASAKAZU NASU \\ Faculty of Engineering, Mie University, Tsu 514, Japan \\ (Received 20 March 1984 and revised 15 June 1984)
}

\begin{abstract}
We define a 'core-matrix' of a transitive sofic subshift, which is unique up to similarity for each transitive sofic subshift. We prove that if there exists a bounded-to-one factor map from one transitive sofic subshift to another, the block of the Jordan form of a core-matrix of this second subshift with non-zero eigenvalues is a principal submatrix of the Jordan form of a core-matrix of the first. We also prove that the subshifts that are almost of finite type are 'spectrally of finite type'.
\end{abstract}

\section{0 . Introduction}

Sofic subshifts (sofic systems), first introduced by Weiss [23], have been studied further by Coven and Paul [4], [5], Fischer [6], [7], Parry [20], Marcus [16] and Boyle [2]. Bounded-to-one factor maps between sofic subshifts and especially those from subshifts of finite type to sofic subshifts, have been studied in their various aspects. It is well known [4], [6] that sofic subshifts are the factors of subshifts of finite type by bounded-to-one factor maps. Further studies of those factor maps are expected to contribute towards a classification of sofic subshifts and towards understanding to what extent sofic subshifts differ from subshifts of finite type, i.e. what is and what is not inherited from subshifts of finite type by bounded-to-one factor maps.

In this paper, we define a 'core-matrix' of a transitive sofic subshift, which is unique up to similarity for each transitive sofic subshift, and extend the result of Kitchens on bounded-to-one factor maps between irreducible subshift of finite type [14] to those between transitive sofic subshifts: we prove that if there exists a bounded-to-one factor map from one transitive sofic subshift to another, the block of the Jordan form of a core-matrix of this second subshift with non-zero eigenvalues is a principal submatrix of the Jordan form of a core-matrix of the first (theorem 5.3). For 1-block factor maps the result is given in a somewhat more definite form (theorem 4.2). We conjecture that transitive sofic subshifts inherit their spectral structure completely, in a sense, from subshifts of finite type; that is, all transitive sofic subshifts are 'spectrally of finite type'. Here, however, we only prove that the subshifts that are 'almost of finite type' in the sense of Marcus [16], are spectrally of finite type $(\$ 6)$.

\section{Background}

Let $A$ be an alphabet (a finite set of symbols) and let $\Omega_{A}=A^{\mathbb{Z}}$. That is,

$$
\Omega_{A}=\left\{\alpha=\left(\cdots \alpha_{-2} \alpha_{-1} \alpha_{0} \alpha_{1} \alpha_{2} \cdots\right) \mid \text { each } \alpha_{i} \in A\right\} .
$$


We define a metric $d$ on $\Omega_{A}$ by $d(\alpha, \beta)=0$ if $\alpha=\beta$ and $d(\alpha, \beta)=1 /(1+k)$ if $\alpha \neq \beta$, where

$$
k=\min \left\{i \in \mathbb{Z}^{+} \mid \alpha_{i} \neq \beta_{i} \text { or } \quad \alpha_{-i} \neq \beta_{-i}\right\} .
$$

With this metric, $\Omega_{A}$ is compact. The shift homeomorphism $\sigma: \Omega_{A} \rightarrow \Omega_{A}$ is defined by $(\sigma(\alpha))_{i}=\alpha_{i+1}, \alpha \in \Omega_{A}, i \in \mathbb{Z}$. The dynamical system $\left(\Omega_{A}, \sigma\right)$ is the full shift over $A$. By a subshift over $A$ we mean a subsystem $(\Omega, \sigma)$ of $\left(\Omega_{A}, \sigma\right)$, where $\Omega$ is a non-empty, closed, $\sigma$-invariant subset of $\Omega_{A}$. (For simplicity, we shall write $\sigma: \Omega \rightarrow \Omega$ instead of $\sigma \mid \Omega: \Omega \rightarrow \Omega$.) Let $\alpha \in \Omega$. A word $x \in A A^{*}$ appears on $\alpha$ if $x=\alpha_{i} \alpha_{i+1} \cdots \alpha_{j}$ for some $i, j \in \mathbb{Z}, i \leq j$, where $A^{*}$ is the free monoid generated by $A$. Let $L(\Omega)$ be the set of all words that appear on some point of $\Omega$. We call $L(\Omega)$ the subshift language of $\Omega$. It is well known and easy to see that a subshift is completely determined by its subshift language. A subset $L$ of $A A^{*}$ will be called a subshift language if $L=L(\Omega)$ for some subshift $(\Omega, \sigma)$ over $A$ or equivalently, if

(i) every subword $x$ with $\lg (x) \geq 1$ of each word in $L$ is also in $L$, and

(ii) for each $x \in L$, there are $y, z \in L$ such that $y x z \in L$.

(For a word $x, \lg (x)$ denotes the length of $x$.) $L$ is said to be transitive if for each pair $y, z \in L$, there exists $x \in L$ such that $y x z \in L$. Clearly, transitivity implies (ii) above. A subshift $(\Omega, \sigma)$ is said to be transitive if $L(\Omega)$ is transitive. It is well known and easy to see that $(\Omega, \sigma)$ is transitive iff it is one-sided topologically transitive (i.e. there exists some $\alpha \in \Omega$ with $\left\{\sigma^{n}(\alpha) \mid n \geq 0\right\}$ dense in $\left.\Omega\right)$. A subshift $(\Omega, \sigma)$ over $A$ is said to be of finite type if there exists a finite set $F \subset A A^{*}$ such that $L(\Omega)=$ $A A^{*}-A^{*} F A^{*}$ and sofic if $L(\Omega)$ is a regular language (in the sense of automata theory, see e.g. [9]). (It appears in [5] that this definition of sofic systems was pointed out by L. W. Goodwyn.)

Let $\left(\Omega_{1}, \sigma_{1}\right)$ and $\left(\Omega_{2}, \sigma_{2}\right)$ be subshifts over alphabets $A$ and $B$, respectively. A continuous map $\pi$ of $\Omega_{1}$ onto $\Omega_{2}$ such that $\pi \sigma_{1}=\sigma_{2} \pi$, is a factor map from $\left(\Omega_{1}, \sigma_{1}\right)$ to $\left(\Omega_{2}, \sigma_{2}\right)$. A mapping $\pi: \Omega_{1} \rightarrow \Omega_{2}$ is called a $k$-block map if there exist $l \in \mathbb{Z}$ and a mapping $f: A^{k} \cap L\left(\Omega_{1}\right) \rightarrow B$ such that $\pi(\alpha)=\beta$ where

$$
\beta_{i}=f\left(\alpha_{i+l} \alpha_{i+l+1} \cdots \alpha_{i+l+k-1}\right) \quad \text { for all } \alpha \in \Omega_{1}, i \in \mathbb{Z} \text {. }
$$

It is well known (Curtis-Hedlund-Lyndon [10]) that a mapping $\pi: \Omega_{1} \rightarrow \Omega_{2}$ is a factor map from $\left(\Omega_{1}, \sigma_{1}\right)$ to $\left(\Omega_{2}, \sigma_{2}\right)$ iff $\pi$ is an onto $k$-block map for some $k \geq 1$.

A non-negative matrix system, abbreviated NMS, of order $m$ over an alphabet $A$, is a system $(A, M, \Phi)$, where $M$ is an $m \times m$ non-negative matrix which is nondegenerate (i.e. has no row or column consisting entirely of zeros), and $\Phi$ is a homomorphism (of monoids) of $A^{*}$ into the monoid consisting of the $m \times m$ non-negative matrices with matrix product such that $\sum_{a \in A} \Phi(a)=M$. Thus $\Phi(\Lambda)$ is the $m \times m$ unit matrix, where $\Lambda$ is the unit element of $A^{*}$, and if $x=a_{1} \cdots a_{m} a_{i} \in A$, then $\Phi(x)=\Phi\left(a_{1}\right) \cdots \Phi\left(a_{r}\right)$. If $M$ is irreducible, then $(A, M, \Phi)$ is called an irreducible NMS, abbreviated INMS.

Let $(A, M, \Phi)$ be an NMS of order $m$. Define $s_{(A, M, \Phi)}: A^{*} \rightarrow \mathbb{R}^{+}$by

$$
s_{(A, M, \Phi)}(x)=\mathbb{J}_{m} \Phi(x) \nabla_{m}^{t} \quad\left(x \in A^{*}\right)
$$

where $\nabla_{m}$ denotes the $m$-dimensional row vector with all entries 1 . (For any matrix 
$N, N^{t}$ denotes the transpose of $N$.) We call $s_{(A, M, \Phi)}$ the standard word-function of $(A, M, \Phi)$. (See [21] for related material.) Let

$$
L_{(A, M, \Phi)}=\left\{x \in A A^{*} \mid s_{(A, M, \Phi)}(x) \neq 0\right\},
$$

which will be called the language generated by $(A, M, \Phi)$. We say that $(A, M, \Phi)$ is normal if there exist positive numbers $c$ and $d$ such that for all $x \in L_{(A, M, \Phi) \text {, }}$ $c \leq s_{(A, M, \Phi)}(x) \leq d$.

An NMS $(A, M, \Phi)$ such that each component of $\Phi(a)$ is an integer for each $a \in A$, can be naturally identified with a directed graph with each arc labelled by some symbol in $A$, which we call a $\lambda$-graph over $A$, or the transition diagram of a non-deterministic finite automaton (see e.g. [9]). That is, $M$ is the adjacency matrix of the directed graph and for each $a \in A, \Phi(a)$ is the adjacency matrix of the subgraph with arcs labelled by $a$. Note that any directed graph is viewed as a $\lambda$-graph with arcs labelled by distinct symbols. For any $\lambda$-graph $(A, M, \Phi)$ and any $x \in A^{*}$, $s_{(A, M, \Phi)}(x)$ is the number of paths generating $x$.

It is easy to see that the language generated by an NMS is a regular subshift language and vice versa. Therefore a subshift $(\Omega, \sigma)$ is sofic iff $L(\Omega)=L_{(A, M, \Phi)}$ for some NMS $(A, M, \Phi)$. Usually sofic subshifts are defined to be the factors (the images of factor maps) of subshifts of finite type. As is well known, they can also be defined to be the images of 1-block maps whose domains are subshifts of finite type described by directed graphs. Each $\lambda$-graph $(A, M, \Phi)$ naturally describes a one-block map of the subshift of finite type described by the directed graph corresponding to $M$ onto the sofic subshift $(\Omega, \sigma)$ such that $L(\Omega)=L_{(A, M, \Phi)}$. For any sofic subshift $(\Omega, \sigma)$, one can choose a right resolving $\lambda$-graph (Shannon graph) as an NMS $(A, M, \Phi)$ with $L(\Omega)=L_{(A, M, \Phi)}[6]$. We say that $(A, M, \Phi)$ is a right resolving $\lambda$-graph [left resolving $\lambda$-graph] if for each $a \in A, \Phi(a)$ is a $0-1$ matrix with at most one 1 in each row [column]. Right [left] resolving $\lambda$-graphs are clearly normal. The following is an immediate interpretation of a result of [6].

Proposition 1.1. Let $(\Omega, \sigma)$ be a sofic subshift over an alphabet. A. The following statements are equivalent.

(1) $(\Omega, \sigma)$ is a transitive sofic subshift.

(2) $L(\Omega)=L_{(A, M, \Phi)}$ for some INMS $(A, M, \Phi)$.

(3) $L(\Omega)=L_{(A, M, \Phi)}$ for some normal INMS $(A, M, \Phi)$.

In fact, Fischer showed in [6] that as a normal INMS $(A, M, \Phi)$ such that $L_{(A, M, \Phi)}=$ $L(\Omega)$ for a transitive sofic subshift $(\Omega, \sigma)$, we can choose a minimal right-resolving $\lambda$-graph, i.e. a right-resolving $\lambda$-graph of the smallest order among all right resolving $\lambda$-graphs generating the same language.

THEOREM 1.2 (Fischer [7]). For any transitive sofic subshift $(\Omega, \sigma)$, the minimal right [left] resolving $\lambda$-graph that generates $L(\Omega)$ is unique.

Standard techniques in finite automata theory, i.e. subset construction and state minimization, give a simple routine to obtain from a given NMS $(A, M, \Phi)$ with $L_{(A, M, \Phi)}$ transitive the minimal right resolving $\lambda$-graph that generates $L_{(A, M, \Phi)}$. 
Let $(A, M, \Phi)$ be an NMS and let $L_{(A, M, \Phi)}$ be transitive. We can assume that $\Gamma=(A, M, \Phi)$ is a $\lambda$-graph (by replacing each non-zero component of the matrices $\Phi(a), a \in A$, by 1). Let $U$ be the set of vertices (states) of $\Gamma$. For a subset $V$ of $U$ and $x \in A^{*}$ we define the $x$-successor of $V$, denoted by $S_{\Gamma}(V, x)$, as the set of all vertices $j$ such that there exists a path going from a vertex in $V$ to $j$ generating $x$ (alternatively, $\left.S_{\mathrm{\Gamma}}(V, x)=\{j \in U \mid \Phi(x)(i, j)>0, i \in V\}\right)$. We define a $\lambda$-graph $\Gamma^{\prime}$ as follows. All the non-empty successors $S_{\Gamma}(U, x), x \in A^{*}$, of $U$ constitute the vertex set of $\Gamma^{\prime}$ and from each vertex $S_{\Gamma}(U, x)$, a unique arc labelled by $a$ goes to $S_{\Gamma}(U, x a)$ if $S_{\Gamma}(U, x a) \neq \varnothing$, for each $a \in A$. Since $S_{\Gamma}(U, x a)=S_{\Gamma}\left(S_{\Gamma}(U, x), a\right)$, an iterative method to generate the successors of $U$ and construct $\Gamma^{\prime}$ is obvious. (It is possible that $\Gamma^{\prime}$ is not non-degenerate.) Clearly $\Gamma^{\prime}$ is right-resolving. Put $s_{0}=U$. Then for each $x \in A A^{*}, x \in L_{(A, M, \Phi)}$ iff $S_{\Gamma^{\prime}}\left(\left\{s_{0}\right\}, x\right) \neq \varnothing$.

By the well-known state minimization routine for finite automata (see e.g. [9]), we can minimize $\Gamma^{\prime}$, if necessary. (See the proof of theorem 6.1.) Therefore we may assume that there exist no distinct equivalent vertices (states) in $\Gamma^{\prime}$, that is $\Gamma^{\prime}$ has no distinct vertices $s_{1}$ and $s_{2}$ such that $S_{\Gamma^{\prime}}\left(\left\{s_{1}\right\}, x\right) \neq \varnothing$ iff $S_{\Gamma^{\prime}}\left(\left\{s_{2}\right\}, x\right) \neq \varnothing$ for all $x \in A^{*}$. Hence by the proof of lemma 2 of [6], there exists a 'magic' word $w$ such that $S_{\Gamma^{\prime}}(S, w)=\{s\}$ for some $s \in S$, where $S$ is the set of all vertices of $\Gamma^{\prime}$. Let $\tilde{\Gamma}=(A, N, \Psi)$ be the maximal strongly connected sub- $\lambda$-graph which contains $s$ ('strongly connected' means that $N$ is irreducible). Since $\tilde{\Gamma}$ is right-resolving and has no distinct equivalent vertices, it follows from lemma 3 of [7] that $\tilde{\Gamma}$ is the minimal right resolving $\lambda$-graph that generates $L_{(A, N, \Psi)}$. Clearly $L_{(A, M, \Phi)} \supset L_{(A, N, \Psi) \text {. }}$ To see that $L_{(A, M, \Phi)} \subset L_{(A, N, \Psi)}$, let $x \in L_{(A, M, \Phi)}$. Since $L_{(A, M, \Phi)}$ is transitive, there exists $y \in L_{(A, M, \Phi)}$ such that $w y x \in L_{(A, M, \Phi)}$. Since $S_{\Gamma}\left(\left\{s_{0}\right\}, w y x\right) \neq \varnothing$ and $S_{\Gamma}\left(\left\{s_{0}\right\}, w\right)=\{s\}$, we have $S_{\tilde{\Gamma}}(\{s\}, y x)=S_{\Gamma^{\prime}}(\{s\}, y x) \neq \varnothing$. Therefore $x$ is generated by $\tilde{\Gamma}$. Thus $L_{(A, N, \Psi)}=$ $L_{(A, M, \Phi)}$ so that $\tilde{\Gamma}=(A, N, \Psi)$ is the desired minimal right resolving $\lambda$-graph that generates $L_{(A, M, \Phi)}$. Note that $\tilde{\Gamma}$ is found as the unique maximal strongly connected sub- $\lambda$-graph of $\Gamma^{\prime}$ with the property that there exists no path going from a vertex of $\tilde{\Gamma}$ to the outside of $\tilde{\Gamma}$, because for each $s^{\prime} \in S$ there exists a path going from $s^{\prime}$ to $s$. (There exists $z \in L_{(A, M, \Phi)}$ with $S_{\Gamma^{\prime}}\left(\left\{s_{0}\right\}, z\right)=\left\{s^{\prime}\right\}$ and $z^{\prime} \in L_{(A, M, \Phi)}$ with $z z^{\prime} w \in$ $L_{(A, M, \Phi)}$ so that $S_{\Gamma^{\prime}}\left(\left\{s^{\prime}\right\}, z^{\prime} w\right)=S_{\Gamma^{\prime}}\left(\left\{s_{0}\right\}, z z^{\prime} w\right)=\{s\}$.)

\section{Canonical word-functions of INMS's}

In this section, we present a fundamental result of this paper in a direct fashion (but see $\S 7$ ).

Let $A$ be an alphabet. For mappings $g$ and $h$ of $A^{*}$ into $\mathbb{R}^{+}$, we write $g \ll h$ if there exists $c>0$ such that $g(x) \leq c h(x)$ for all $x \in A A^{*}$. If $g \ll h$ and $h \ll g$, then we write $g \sim h$.

Let $(A, M, \Phi)$ be an INMS of order $m$. Since $M$ is an irreducible non-negative matrix, by the Perron-Frobenius theorem (see [8]) there exists a positive eigenvalue $\lambda$ such that $\left|\lambda^{\prime}\right| \leq \lambda$ for any eigenvalue $\lambda^{\prime}$ of $M$, and moreover eigenvectors corresponding to $\lambda$ have strictly positive entries. We denote that maximal eigenvalue $\lambda$ of $M$ by $\lambda_{M}$. Let $u_{M}=\left(u_{1}, \ldots, u_{m}\right)$ be the left eigenvector corresponding to $\lambda_{M}$ with $\sum_{i=1}^{m} u_{i}=1$ and let $v_{M}=\left(v_{1}, \ldots, v_{m}\right)^{\prime}$ be the right eigenvector corresponding to $\lambda_{M}$ 
such that $\sum_{i=1}^{m} u_{i} v_{i}=1$. We define $c_{(A, M, \Phi)}: A^{*} \rightarrow \mathbb{R}^{+}$by

$$
c_{(A, M, \Phi)}(x)=u_{M} \Phi(x) v_{M} \quad\left(x \in A^{*}\right) .
$$

We call $c_{(A, M, \Phi)}$ the canonical word-function of the INMS $(A, M, \Phi)$. Note that $c_{(A, M, \Phi)} \sim s_{(A, M, \Phi)}$.

LeMmA 2.1. Let $(A, M, \Phi)$ and $(A, N, \Psi)$ be INMS's with $c_{(A, M, \Phi)} \sim c_{(A, N, \Psi)}$. Then $\lambda_{M}=\lambda_{N}$

Proof. It suffices to show that if $c_{(A, M, \Phi)} \ll c_{(A, N, \Psi)}$, then $\lambda_{M} \leq \lambda_{N}$. Assume that there exists a number $c>0$ such that $u_{M} \Phi(x) v_{M} \leq c u_{N} \Psi(x) v_{N}$ for all $x \in A^{*}$. Recalling that $\sum_{a \in A} \Phi(a)=M$, we have

$$
\begin{aligned}
\lambda_{M}^{i} & =u_{M} M^{i} v_{M}=u_{M}\left(\sum_{x \in A^{i}} \Phi(x)\right) v_{M} \\
& \leq c u_{N}\left(\sum_{x \in A^{i}} \Psi(x)\right) v_{N}=c u_{N} N^{i} v_{N}=c \lambda_{N}^{i},
\end{aligned}
$$

for all $i \in \mathbb{Z}^{+}$. Thus $\lambda_{M} \leq \lambda_{N}$.

LeMma 2.2. Let $(A, M, \Phi)$ be an INMS with $\lambda_{M}=1$. Then for any $y, z \in A^{*}$,

$$
\lim _{n \rightarrow \infty} \frac{1}{n} \sum_{i=0}^{n-1} \sum_{x \in A^{i}} c_{(A, M, \Phi)}(y x z)=c_{(A, M, \Phi)}(y) c_{(A, M, \Phi)}(z) .
$$

Proof. Let $v_{M}=\left(v_{1}, \ldots, v_{m}\right)^{t}$. Let $D$ be the $m \times m$ diagonal matrix such that $D(i, i)=$ $v_{i}, i=1, \ldots, m$. Let $P=D^{-1} M D$ and let $\Pi(a)=D^{-1} \Phi(a) D$ for each $a \in A$. Then $P$ is a stochastic matrix (with each row sum 1 ) and we have an INMS $(A, P, \Pi)$. It is easy to see that $u_{P}=\left(u_{1} v_{1}, \ldots, u_{m} v_{m}\right), v_{P}=\mathbb{1}_{m}^{t}$, and hence $c_{(A, M, \Phi)}=c_{(A, P, I)}$. Since $P$ is an irreducible stochastic matrix, it is well known that

$$
\lim _{n \rightarrow \infty} \frac{1}{n} \sum_{i=0}^{n-1} P^{i}=\nabla_{m}^{t} u_{P}
$$

Let $y, z \in A^{*}$. Then, using the above we have

$$
\begin{aligned}
\frac{1}{n} \sum_{i=0}^{n-1} \sum_{x \in A^{i}} c_{(A, M, \Phi)}(y x z) & =\frac{1}{n} \sum_{i=0}^{n-1} \sum_{x \in A^{i}} u_{P} \Pi(y) \Pi(x) \Pi(z) \nabla_{m}^{\prime} \\
& =\frac{1}{n} \sum_{i=0}^{n-1} u_{P} \Pi(y) P^{i} \Pi(z) \nabla_{m}^{t}=u_{P} \Pi(y)\left(\frac{1}{n} \sum_{i=0}^{n-1} P^{i}\right) \Pi(z) \nabla_{m}^{t} \\
& \rightarrow u_{P} \Pi(y) \mathbb{1}_{m}^{t} u_{P} \Pi(z) \mathbb{1}_{m}^{t}=c_{(A, M, \Phi)}(y) c_{(A, M, \Phi)}(z) .
\end{aligned}
$$

THEOREM 2.3. Let $(A, M, \Phi)$ and $(A, N, \Psi)$ be INMS's with $\lambda_{M}=\lambda_{N}$. If $c_{(A, M, \Phi)} \ll$ $c_{(A, N, \Psi)}$, then $c_{(A, M, \Phi)}=c_{(A, N, \Psi)}$.

Proof. Put $g=c_{(A, M, \Phi)}$ and $h=c_{(A, N, \Psi)}$. It suffices to show that

$\left(^{*}\right)$ if $g \ll h$, then $g(x) \geq h(x)$ for all $x \in A^{*}$.

For the conclusion of $\left(^{*}\right)$ implies $g \gg h$ and hence again by $\left({ }^{*}\right), g(x) \leq h(x)$ for all $x \in A^{*}$. We may assume that $\lambda_{M}=\lambda_{N}=1$, because we can replace $M$, the $\Phi(a)$ 's, $N$, and the $\Psi(a)$ 's by those divided by $\lambda=\lambda_{M}=\lambda_{N}$. since $g \ll h$, there exists

$$
d=\sup \left\{g(x) / h(x) \mid x \in L_{(A, N, \Psi)}\right\} \text {. }
$$


Let $\varepsilon>0$. Then there exists $y_{\varepsilon} \in L_{(A, M, \Phi)}$ such that

$$
g\left(y_{\varepsilon}\right) / h\left(y_{\varepsilon}\right) \geq d-\varepsilon \text {. }
$$

Since $\lambda_{M}=\lambda_{N}=1$, it follows that for each $k \in \mathbb{Z}^{+}$,

$$
\sum_{x \in A^{k}} g\left(y_{\varepsilon} x\right)=g\left(y_{\varepsilon}\right) \text { and } \sum_{x \in A^{k}} h\left(y_{\varepsilon} x\right)=h\left(y_{\varepsilon}\right) .
$$

Therefore, using (2.1), we have, for each $k \in \mathbb{Z}^{+}$,

$$
\sum_{x \in A^{k}}\left(d h\left(y_{\varepsilon} x\right)-g\left(y_{\varepsilon} x\right)\right) \leq \varepsilon h\left(y_{\varepsilon}\right)
$$

Let $z$ be any word in $A^{*}$ and let $k=\lg (z)$. Using lemma 2.2 and (2.2) and noting that $d h\left(y_{F} x\right)-g\left(y_{\varepsilon} x\right) \geq 0$ for all $x \in A^{*}$, we have

$$
\begin{aligned}
d h\left(y_{\varepsilon}\right) h(z)-g\left(y_{\varepsilon}\right) g(z) & =\lim _{n \rightarrow \infty} \frac{1}{n} \sum_{i=0}^{n-1} \sum_{x \in A^{i}}\left(d h\left(y_{\varepsilon} x z\right)-g\left(y_{\varepsilon} x z\right)\right) \\
& \leq \lim _{n \rightarrow \infty} \frac{1}{n} \sum_{i=0}^{n-1} \sum_{x \in A^{i+k}}\left(d h\left(y_{\varepsilon} x\right)-g\left(y_{\varepsilon} x\right)\right) \leq \varepsilon h\left(y_{\varepsilon}\right) .
\end{aligned}
$$

Hence

$$
h(z) \leq \frac{1}{d}\left(\varepsilon+\left(g\left(y_{\varepsilon}\right) / h\left(y_{\varepsilon}\right)\right) g(z)\right) \leq \frac{1}{d}(\varepsilon+d g(z))=\varepsilon / d+g(z) .
$$

Since $\varepsilon$ is arbitrarily small, $h(z) \leq g(z)$. Thus we have shown $\left(^{*}\right)$.

Corollary 2.4. Let $(A, M, \Phi)$ and $(A, N, \Psi)$ be normal INMS's with $L_{(A, M, \Phi)}=$

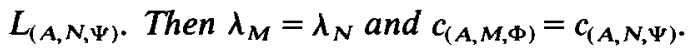

Proof. By lemma 2.1 and the above theorem.

Corollary 2.5. Let $(A, M, \Phi)$ and $(A, N, \Psi)$ be INMS's with $\lambda_{M}=\lambda_{N}$. Then $s_{(A, M, \Phi)} \ll s_{(A, N, \Psi)}$ iff $s_{(A, M, \Phi)} \gg s_{(A, N, \Psi) \text {. }}$

\section{Core-matrices of transitive sofic subshifts}

First we need some results on 'matrix systems with specified initial and final vectors' (linear (space) automata) which were developed by Matuura, Inagaki, and Fukumura [17] and Inagaki, Fukumura, and Matuura [11].

A matrix system, abbreviated MS, $(A, M, \Phi)$ of order $m$ is the same as an NMS except that each entry of $M$ and $\Phi(a), a \in A$, may be a (not necessarily non-negative) real number and $M$ may be degenerate. We consider an $\operatorname{MS}(A, M, \Phi)$ with specified initial and final vectors $u$ and $v$, where $u$ and $v$ are $m$-dimensional row and column vectors with real entries, respectively, if the order of the MS is $m$. It will be simply referred to as an $\operatorname{MS}(A, M, \Phi)$ with $(u, v)$. We say that one $\operatorname{MS}(A, M, \Phi)$ with $(u, v)$ and another $\operatorname{MS}(A, N, \Psi)$ with $\left(u^{\prime}, v^{\prime}\right)$ are equivalent if

$$
u \Phi(x) v=u^{\prime} \Psi(x) v^{\prime} \quad \text { for all } x \in A^{*} \text {. }
$$

An MS $(A, M, \Phi)$ of order $m$ with $(u, v)$ is row-reduced if $\left\{u \Phi(x) \mid x \in A^{*}\right\}$ generates the row-vector space $\mathbb{R}^{m}$ and column-reduced if $\left\{\Phi(x) v \mid x \in A^{*}\right\}$ generates the columnvector space $\mathbb{R}^{m}$. It is reduced if it is both row-reduced and column-reduced. (Note: as is well known and easily seen, $\left\{u \Phi(x) \mid x \in \bigcup_{i=0}^{m-1} A^{i}\right\}$ and $\left\{u \Phi(x) \mid x \in A^{*}\right\}$ generate 
the same subspace and so do $\left\{\Phi(x) v \mid x \in \bigcup_{i=0}^{m-1} A^{i}\right\}$ and $\left\{\Phi(x) v \mid x \in A^{*}\right\}$.) An MS $(\bar{A}, \bar{M}, \bar{\Phi})$ with $(\bar{u}, \bar{v})$ is called a reduced form of an $\operatorname{MS}(A, M, \Phi)$ with $(u, v)$ if the former is reduced and equivalent to the latter. A construction of a reduced form of a given MS $(A, M, \Phi)$ with $(u, v)$ appears in [17]. (It is also contained in [11].) For our purposes, however, we give a different construction.

Construction of a reduced form. For an $\operatorname{MS}(A, M, \Phi)$ of order $m$ with $(u, v)$, a reduced form of it is given as follows. Let $U$ be the subspace of the vector space $\mathbb{R}^{m}$ generated by $\left\{u \Phi(x) \mid x \in A^{*}\right\}$. Then $U$ is an invariant subspace of $\Phi(a)$ for each $a \in A$. Let $k=\operatorname{dim} U$ and let $L$ be a $k \times m$ matrix whose rows form a basis of $U$. Then there exist $k \times k$ matrices $\hat{\Phi}(a), a \in A$, such that $L \Phi(a)=\hat{\Phi}(a) L$ for all $a \in A$. Then it is easy to see that the $\operatorname{MS}(A, \hat{M}, \hat{\Phi})$ with $(\hat{u}, \hat{v})$ is equivalent to $(A, M, \Phi)$ with $(u, v)$, where $\hat{M}=\sum_{a \in A} \hat{\Phi}(a)$, $\hat{u}$ is the $k$-dimensional row vector with $\hat{u} L=u$ and $\hat{v}=L v$. We have $L M=\hat{M} L$. The subspace $\hat{U}$ of $\mathbb{R}^{k}$ generated by $\left\{\hat{u} \hat{\Phi}(x) \mid x \in A^{*}\right\}$ is equal to $\mathbb{R}^{k}$ because $\hat{U} L=U$, the rank of $L$ is $k$ and $\operatorname{dim} U=k$. Let $\hat{V}$ be the subspace of $\mathbb{R}^{k}$ generated by $\left\{\hat{\Phi}(x) \hat{v} \mid x \in A^{*}\right\}$. Then $\hat{V}$ is an invariant subspace of $\hat{\Phi}(a)$ for each $a \in A$. Let $l=\operatorname{dim} \hat{V}$ and let $R$ be a $k \times l$ matrix whose columns form a basis of $\hat{V}$. Then there exist $l \times l$ matrices $\bar{\Phi}(a), a \in A$, such that $\hat{\Phi}(a) R=R \bar{\Phi}(a)$ for all $a \in A$. It is easy to see that $\operatorname{MS}(A, \bar{M}, \bar{\Phi})$ with $(\bar{u}, \bar{v})$ is equivalent to $(A, \hat{M}, \hat{\Phi})$ with $(\hat{u}, \hat{v})$, where $\bar{M}=\sum_{a \in A} \bar{\Phi}(a), \bar{u}=\hat{u} R$ and $\bar{v}$ is the $l$-dimensional column vector with $\hat{v}=R \bar{v}$. We have $\hat{M} R=R \bar{M}$. The subspace $\bar{V}$ of $\mathbb{R}^{l}$ generated by $\left\{\bar{\Phi}(x) \bar{v} \mid x \in A^{*}\right\}$ equals $\mathbb{R}^{l}$ because $R \bar{V}=\hat{V}$, the rank of $R$ is $l$ and $\operatorname{dim} \hat{V}=l$. Moreover the subspace $\bar{U}$ of $\mathbb{R}^{\prime}$ generated by $\left\{\bar{u} \bar{\Phi}(x) \mid x \in A^{*}\right\}$ has dimension $l$ because $\bar{U}=\hat{U} R=\mathbb{R}^{k} R$ and $R$ has rank $l$. Therefore, $(A, \bar{M}, \bar{\Phi})$ with $(\bar{u}, \bar{v})$ is reduced. Since it is equivalent to $(A, M, \Phi)$ with $(u, v)$, it is a desired reduced form.

We recall that $L \Phi(a)=\hat{\Phi}(a) L$ for each $a \in A, L M=\hat{M} L, \hat{\Phi}(a)(a \in A)$ and $\hat{M}$ are $k \times k$ matrices, and $L$ has rank $k$. We also recall that $\hat{\Phi}(a) R=R \bar{\Phi}(a)$ for each $a \in A, \hat{M} R=R \bar{M}, \bar{\Phi}(a)(a \in A)$ and $\bar{M}$ are $l \times l$ matrices and $R$ has rank $l$. Thus the Jordan form of $\bar{\Phi}(a)$ is a principal submatrix of that of $\Phi(a)$ for each $a \in A$, and the Jordan form of $\bar{M}$ is a principal submatrix of that of $M$.

A result similar to the following appears in [11]. We shall give a direct proof for completeness.

THEOREM 3.1. Let an MS $(A, M, \Phi)$ with $(u, v)$ be reduced and be equivalent to an MS $(A, N, \Psi)$ with $\left(u^{\prime}, v^{\prime}\right)$. Then the Jordan form of $\Phi(a)$ is a principal submatrix of that of $\Psi(a)$ for each $a \in A$ and the Jordan form of $M$ is a principal submatrix of that of $N$.

Proof. By the above (consider a reduced form of $(A, N, \Psi)$ with $\left(u^{\prime}, v^{\prime}\right)$ ), it suffices to show that if $(A, M, \Phi)$ with $(u, v)$ and $(A, N, \Psi)$ with $\left(u^{\prime}, v^{\prime}\right)$ are equivalent and both of them are reduced, then there exists a non-singular matrix $T$ such that $\Phi(a) T=T \Psi(a)$ for all $a \in A$ and $M T=T N$.

Let $k$ and $l$ be the orders of $(A, M, \Phi)$ and $(A, N, \Psi)$, respectively, and we assume without loss of generality that $k \geq l$. Since $\mathbb{R}^{k}$ is generated by $\left\{u \Phi(x) \mid x \in A^{*}\right\}$, there exist $x_{1}, \ldots, x_{k} \in A^{*}$ such that $u \Phi\left(x_{1}\right), \ldots, u \Phi\left(x_{k}\right)$ form a basis of $\mathbb{R}^{k}$. Let $T: \mathbb{R}^{k} \rightarrow \mathbb{R}^{l}$ be the linear map such that $T\left(u \Phi\left(x_{i}\right)\right)=u^{\prime} \Psi\left(x_{i}\right), i=1, \ldots, k$. Since $(A, M, \Phi)$ with 
$(u, v)$ and $(A, N, \Psi)$ with $\left(u^{\prime}, v^{\prime}\right)$ are equivalent,

$$
\left(c_{1} u \Phi\left(x_{1}\right)+\cdots+c_{k} u \Phi\left(x_{k}\right)\right) \Phi(x) v=\left(c_{1} u^{\prime} \Psi\left(x_{1}\right)+\cdots+c_{k} u^{\prime} \Psi\left(x_{k}\right)\right) \Psi(x) v^{\prime},
$$

for any $c_{1}, \ldots, c_{k} \in \mathbb{R}$ and any $x \in A^{*}$. This implies that

(*) for each $w \in \mathbb{R}^{k}, w \Phi(x) v=T(w) \Psi(x) v^{\prime}$ for all $x \in A^{*}$.

Thus if $T(w)=0$, then $w \Phi(x) v=0$ for all $x \in A^{*}$ so that $w=0$ because $\left\{\Phi(x) v \mid x \in A^{*}\right\}$ generates $\mathbb{R}^{k}$. Therefore $T$ is one-to-one and $l \geq k$. Hence $l=k$ and $T$ is an isomorphism. Moreover, it follows from (*) that for any $w \in \mathbb{R}^{k}, a \in A$, and $x \in A^{*}$,

$$
T(w \Phi(a)) \Psi(x) v^{\prime}=w \Phi(a) \Phi(x) v=T(w) \Psi(a) \Psi(x) v^{\prime}
$$

Since $\left\{\Psi(x) v^{\prime} \mid x \in A^{*}\right\}$ generates $\mathbb{R}^{k}$, we have $T(w \Phi(a))=T(w) \Psi(a)$ for all $w \in \mathbb{R}^{k}$ and $a \in A$. Thus there exists a non-singular $k \times k$ matrix such that $\Phi(a) T=T \Psi(a)$ for all $a \in A$ and hence $M T=T N$.

Let $(A, M, \Phi)$ be an INMS. Let $(A, \bar{M}, \bar{\Phi})$ with $(\bar{u}, \bar{v})$ be a reduced form of $(A, M, \Phi)$ with $\left(u_{M}, v_{M}\right)$. Then $(A, \bar{M}, \bar{\Phi})$ is called a core-MS of $(A, M, \Phi)$ and $\bar{M}$ is called a core-matrix of $(A, M, \Phi)$.

Proposition 3.2. Let $(A, M, \Phi)$ and $(A, N, \Psi)$ be INMS's with $s_{(A, M, \Phi)} \sim s_{(A, N, \Psi)}$. Let $(A, \bar{M}, \bar{\Phi})$ and $(A, \bar{N}, \bar{\Psi})$ be their core-MS's, respectively. Then $(A, \bar{M}, \bar{\Phi})$ and $(A, \bar{N}, \bar{\Psi})$ are similar, i.e. there exists a non-singular matrix $T$ with $\bar{\Phi}(a) T=T \bar{\Psi}(a)$ for all $a \in A$. In particular $\bar{M}$ and $\bar{N}$ are similar. The Jordan form of $\bar{M}$ is a principal submatrix of that of $N$ and $\lambda_{M}$ is an eigenvalue of $\bar{M}$.

Proof. If $(A, \bar{M}, \bar{\Phi})$ with $(\bar{u}, \bar{v})$ is the reduced form of $(A, M, \Phi)$ with $\left(u_{M}, v_{M}\right)$ obtained by the previous construction, then $\hat{u} \hat{M}=\lambda_{M} \hat{u}$, since

$$
\hat{u} \hat{M} L=\hat{u} L M=u_{M} M=\lambda_{M} u_{M}=\lambda_{M} \hat{u} L
$$

and $L$ is a $k \times m$ matrix of rank $k$. Hence

$$
\bar{u} \bar{M}=\hat{u} R \bar{M}=\hat{u} \hat{M} R=\lambda_{M} \hat{u} R=\lambda_{M} \bar{u} .
$$

Thus $\lambda_{M}$ is an eigenvalue of $\bar{M}$. The remainder follows from lemma 2.1 , theorem 2.3 , and theorem 3.1 .

Let $(\Omega, \sigma)$ be a transitive sofic subshift. Let $(A, M, \Phi)$ be a normal INMS such that $L_{(A, M, \Phi)}=L(\Omega)$. A core-MS $(A, \bar{M}, \bar{\Phi})$ of $(A, M, \Phi)$ is called a core-MS of $(\Omega, \sigma)$ and $\bar{M}$ is called a core-matrix of $(\Omega, \sigma)$.

As mentioned in $\S 1$, we can obtain a minimal right resolving $\lambda$-graph as a normal INMS $(A, M, \Phi)$ with $L_{(A, M, \Phi)}=L(\Omega)$ for any transitive sofic subshift $(\Omega, \sigma)$. Therefore the following proposition would be useful to calculate a core-MS of a transitive sofic subshift.

PRoposition 3.3. If an INMS $(A, M, \Phi)$ is a minimal right resolving $\lambda$-graph, then it is row-reduced.

Proof. Since the right resolving $\lambda$-graph $(A, M, \Phi)$ is minimal, it has a magic word $w \in A A^{*}$, by the proof of lemma 2 of [6]. That is, $u_{M} \Phi(w)$ has a unique non-zero entry. Since $(A, M, \Phi)$ is right-resolving and irreducible, for each $1 \leq i \leq m$ there exists $x_{i} \in A^{*}$ such that $u_{M} \Phi\left(w x_{i}\right)$ has a non-zero component only in the $i$ th entry, where $m$ is the order of $(A, M, \Phi)$. Thus $(A, M, \Phi)$ is row-reduced. 
Remark. An INMS which is a minimal right resolving $\lambda$-graph, is not always a core-MS (of itself) (see $\S 7$ ).

\section{An invariant for bounded-to-one 1-block factor maps}

Let $\left(\Omega_{1}, \sigma_{1}\right)$ and $\left(\Omega_{2}, \sigma_{2}\right)$ be transitive sofic subshifts over alphabets $A \subset L\left(\Omega_{1}\right)$ and $B \subset L\left(\Omega_{2}\right)$, respectively. Let $\pi: \Omega_{1} \rightarrow \Omega_{2}$ be a 1 -block map. Then there exist $l \in \mathbb{Z}$ and a mapping $f: A \rightarrow B$ such that $\pi(\alpha)=\beta$ where $\beta_{i}=f\left(\alpha_{i+l}\right)$ for all $\alpha \in \Omega_{1}, i \in \mathbb{Z}$. Let $f^{*}: L\left(\Omega_{1}\right) \rightarrow L\left(\Omega_{2}\right)$ be defined by

$$
f^{*}\left(a_{1}, \ldots a_{r}\right)=f\left(a_{1}\right) \cdots f\left(a_{r}\right) \quad \text { for } a_{1} \cdots a_{r} \in L\left(\Omega_{1}\right) \text { with } a_{i} \in A \text {. }
$$

LEMMA 4.1. $\pi$ is bounded-to-one iff $f^{*}$ is bounded-to-one, and moreover $\pi$ is onto iff $f^{*}$ is onto.

Proof. This is proved by a straightforward modification of the proof of proposition 2 of [18] using the obvious generalization of proposition 1 of [18] to $\lambda$-graphs.

Let $(A, M, \Phi)$ be a normal INMS with $L\left(\Omega_{1}\right)=L_{(A, M, \Phi)}$ and $(B, N, \Psi)$ a normal INMS with $L\left(\Omega_{2}\right)=L_{(B, N, \Psi)}$. We define an INMS $\left(B, M, \Phi_{f}\right)$ by

$$
\Phi_{f}(b)=\sum_{a \in f^{-1}(b)} \Phi(a) \quad(b \in B)
$$

It is easy to see that

$$
c_{\left(B, M, \Phi_{f}\right)}(y)=\sum_{x \in\left(f^{*}\right)^{-1}(y)} c_{(A, M, \Phi)}(x) \quad\left(y \in L\left(\Omega_{2}\right)\right) .
$$

Since $(A, M, \Phi)$ and $(B, N, \Psi)$ are normal, $c_{(A, M, \Phi)} \sim \chi_{L_{(A, M, \Phi)}}$ and $c_{(B, N, \Psi)} \sim \chi_{L_{(B, N, \Psi)}}$. Therefore it follows from (4.1) that $f^{*}$ is bounded-to-one iff $c_{\left(B, M, \Phi_{f}\right)} \ll c_{(B, N, \Psi)}$ and moreover, $f^{*}$ is onto iff $c_{\left(B, M, \Phi_{f}\right)} \gg c_{(B, N, \Psi)}$. Thus it follows from corollary 2.5 that if $\lambda_{M}=\lambda_{N}$, then $f^{*}$ is onto iff $f^{*}$ is bounded-to-one. (This is a generalization of theorem 3 of [18].) Combining this with lemma 4.1 we conclude that if $\lambda_{M}=\lambda_{N}$, then $\pi$ is onto iff $\pi$ is bounded-to-one. Hence we have presented another proof of a result of Coven and Paul in [4].

Suppose that $\pi$ is bounded-to-one and onto. Then $f^{*}$ is bounded-to-one and onto so that $c_{\left(B, M, \Phi_{f}\right)} \sim c_{(B, N, \Psi)}$. Hence by corollary $2.4, c_{\left(B, M, \Phi_{f}\right)}=c_{(B, N, \Psi)}$. Thus from (4.1),

$$
c_{(B, N, \Psi)}(y)=\sum_{x \in\left(f^{*}\right)^{-1}(y)} c_{(A, M, \Phi)}(x) \quad\left(y \in L_{(B, N, \Psi)}\right)
$$

Let $(A, \bar{M}, \bar{\Phi})$ be a core-MS of $(A, M, \Phi)$ and let $(B, \bar{N}, \bar{\Psi})$ be that of $(B, N, \Psi)$. There exist $u_{\bar{M}}$ and $v_{\bar{M}}$ such that

$$
c_{(A, M, \Phi)}(x)=u_{\bar{M}} \bar{\Phi}(x) v_{\bar{M}} \quad \text { for all } x \in A^{*},
$$

and also there exist $u_{\bar{N}}$ and $v_{\bar{N}}$ such that

$$
c_{(B, N, \Psi)}(y)=u_{\bar{N}} \bar{\Psi}(y) v_{\bar{N}} \quad \text { for all } y \in A^{*} .
$$

Let $i^{*}: L_{(B, N, \Psi)} \rightarrow B^{*}$ be the canonical injection and let $g^{*}=i^{*} f^{*}$. Let $(B, \bar{M}, Y)$ be the MS defined by $Y(b)=\sum_{a \in\left(g^{*}\right)^{-1}(b)} \bar{\Phi}(a), b \in B$. Then it is easy to see that for all $y \in B^{*}$,

$$
u_{\bar{M}} \Upsilon(y) v_{\bar{M}}=\sum_{x \in\left(g^{*}\right)^{-1}(y)} u_{\bar{M}} \bar{\Phi}(x) v_{\bar{M}}
$$


Hence, using (4.2), we have

$$
u_{\bar{M}} Y(y) v_{\bar{M}}=\sum_{x \in\left(g^{*}\right)^{-1}(y)} c_{(A, M, \Phi)}(x)=c_{(B, N, \Psi)}(y)=u_{\bar{N}} \bar{\Psi}(y) v_{\bar{N}},
$$

for all $y \in B^{*}$. Therefore, $(B, \bar{M}, Y)$ with $\left(u_{\bar{M}}, v_{\bar{M}}\right)$ is equivalent to $(B, \bar{N}, \bar{\Psi})$ with $\left(u_{\bar{N}}, v_{\bar{N}}\right)$. Since the latter is reduced, it follows from theorem 3.1 that the Jordan form of $\bar{N}$ is a principal submatrix of the Jordan form of $\bar{M}$. Thus we have proved the following.

THEOREM 4.2. Let $\left(\Omega_{1}, \sigma_{1}\right)$ and $\left(\Omega_{2}, \sigma_{2}\right)$ be transitive sofic subshifts. If there exists a bounded-to-one 1-block map of $\Omega_{1}$ onto $\Omega_{2}$, then the Jordan form of a core-matrix $\bar{N}$ of $\left(\Omega_{2}, \sigma_{2}\right)$ is a principal submatrix of the Jordan form of a core-matrix $\bar{M}$ of $\left(\Omega_{1}, \sigma_{1}\right)$.

\section{An invariant for bounded-to-one factor maps}

To extend theorem 4.2 to $k$-block maps for any $k$ we need some technical results on $k$-block MS's introduced in the following.

Let $(A, M, \Phi)$ be an MS of order $m$ with $(u, v)$. For each $a \in A$, let

$$
J_{a}=\{(i, j, a) \mid i, j=1, \ldots, m, \Phi(a)(i, j) \neq 0\} .
$$

Let $J=\bigcup_{a \in A} J_{a}$. We define matrices $\Gamma(a), a \in A$, and $\Gamma$ indexed by $\{1, \ldots, m\}$ and $J$ as follows: for each $a \in A$,

$$
\Gamma(a)\left(i^{\prime},\left(i, j, a^{\prime}\right)\right)= \begin{cases}\Phi(a)(i, j) & \text { if } i^{\prime}=i \text { and } a^{\prime}=a, \\ 0 & \text { otherwise, }\end{cases}
$$

and $\Gamma=\sum_{a \in A} \Gamma(a)$. We also define matrices $\Delta(a), a \in A$, and $\Delta$ indexed by $J$ and $\{1, \ldots, m\}$ as follows: for each $a \in A$,

$$
\Delta(a)\left(\left(i, j, a^{\prime}\right), j^{\prime}\right)= \begin{cases}1 & \text { if } j^{\prime}=j \text { and } a^{\prime}=a, \\ 0 & \text { otherwise, }\end{cases}
$$

and $\Delta=\sum_{a \in A} \Delta(a)$. Then we observe that for $a, b \in A$,

$$
\Gamma(a) \Delta(b)= \begin{cases}\Phi(a) & \text { if } a=b, \\ 0 & \text { if } a \neq b,\end{cases}
$$

Hence we have

$$
\Gamma \Delta=M
$$

Let

$$
M^{\prime}=\Delta \Gamma,
$$

and for each pair $a, b \in A$, let

$$
\Phi^{\prime}(a b)=\Delta(a) \Gamma(b),
$$

let $u^{\prime}=u \Gamma$ and let $v^{\prime}=\Delta v$. Since $M^{\prime}=\sum_{a, b \in A} \Delta(a) \Gamma(b)=\sum_{w \in A^{2}} \Phi^{\prime}(w)$, we obtain an MS $\left(A^{2}, M^{\prime}, \Phi^{\prime}\right)$ with $\left(u^{\prime}, v^{\prime}\right)$. For any word $\left(a_{1} b_{1}\right)\left(a_{2} b_{2}\right) \cdots\left(a_{r} b_{r}\right)$ in $A^{2}\left(A^{2}\right)^{*}$ with $a_{i}, b_{i} \in A, i=1, \ldots, r$,

$$
\begin{aligned}
& u^{\prime} \Phi^{\prime}\left(\left(a_{1} b_{1}\right) \cdots\left(a_{r} b_{r}\right)\right) v^{\prime} \\
& \quad=u^{\prime} \Phi^{\prime}\left(\left(a_{1} b_{1}\right)\right) \cdots \Phi^{\prime}\left(\left(a_{r} b_{r}\right)\right) v^{\prime}=u \Gamma \Delta\left(a_{1}\right) \Gamma\left(b_{1}\right) \cdots \Delta\left(a_{r}\right) \Gamma\left(b_{r}\right) \Delta v \\
& \quad=u\left(\sum_{a \in A} \Gamma(a)\right) \Delta\left(a_{1}\right) \Gamma\left(b_{1}\right) \Delta\left(a_{2}\right) \cdots \Gamma\left(b_{r-1}\right) \Delta\left(a_{r}\right) \Gamma\left(b_{r}\right)\left(\sum_{b \in B} \Delta(b)\right) v .
\end{aligned}
$$


Hence, from (5.1), we have

$$
u^{\prime} \Phi^{\prime}\left(\left(a_{1} b_{1}\right) \cdots\left(a_{r} b_{r}\right)\right) v^{\prime}= \begin{cases}u \Phi\left(a_{1} \cdots a_{r} b_{r}\right) v & \text { if } b_{i}=a_{i+1} \text { for } i=1, \ldots, r-1, \\ 0 & \text { otherwise. }\end{cases}
$$

For each integer $k \geq 1$, we define an $\operatorname{MS}\left(A_{k}, M_{k}, \Phi_{k}\right)$ with $\left(u_{k}, v_{k}\right)$ as follows:

$$
\left(A_{1}, M_{1}, \Phi_{1}\right)=(A, M, \Phi) \text { and }\left(u_{1}, v_{1}\right)=(u, v) \text {; }
$$

for $k \geq 2,\left(A_{k}, M_{k}, \Phi_{k}\right)=\left(\left(A_{k-1}\right)^{2},\left(M_{k-1}\right)^{\prime},\left(\Phi_{k-1}\right)^{\prime}\right)$ and $\left(u_{k}, v_{k}\right)=\left(\left(u_{k-1}\right)^{\prime},\left(v_{k-1}\right)^{\prime}\right)$.

We define an injection $\tau_{k}:\left(A^{k}\right)^{*} \rightarrow\left(A_{k}\right)^{*}$ and a bijection $\theta_{k}: A^{k} A^{*} \rightarrow A^{k}\left(A^{k}\right)^{*}$ for each $k \geq 1$ as follows: $\tau_{1}$ is the identity mapping and for $k \geq 2, \tau_{k}$ is the homomorphism of free monoids determined by

$$
\tau_{k}\left(a_{1} \cdots a_{k}\right)=\left(\tau_{k-1}\left(a_{1} \cdots a_{k-1}\right) \tau_{k-1}\left(a_{2} \cdots a_{k}\right)\right) \quad\left(a_{1}, \ldots, a_{k} \in A\right),
$$

and for $a_{1}, \ldots, a_{n} \in A, n \geq k$,

$$
\theta_{k}\left(a_{1} \cdots a_{n}\right)=\left(a_{1} \cdots a_{k}\right)\left(a_{2} \cdots a_{k+1}\right) \cdots\left(a_{n-k+1} \cdots a_{n}\right) \text {. }
$$

LEMMA 5.1. For each $k \geq 1$, the following statements hold.

(1) The block of the Jordan form of $M_{k}$ with non-zero eigenvalues is identical to that of $M$.

(2) $u_{k} \Phi_{k}\left(\tau_{k} \theta_{k}(x)\right) v_{k}=u \Phi(x) v$ for $x \in A^{k} A^{*}$ and if $z \in A_{k}\left(A_{k}\right)^{*}-\tau_{k} \theta_{k}\left(A^{k} A^{*}\right)$, then $u_{k} \Phi_{k}(z) v_{k}=0$.

(3) $\Phi_{k}(c)=0$ for all $c \in A_{k}-\tau_{k} \theta_{k}\left(A^{k}\right)=A_{k}-\tau_{k}\left(A^{k}\right)$.

(4) If $u$ [v] is a row [column] eigenvector corresponding to an eigenvalue $\lambda$ of $M$, then $u_{k}\left[v_{k}\right.$ is a row [column] eigenvector corresponding to $\lambda$.

(5) If $(A, M, \Phi)$ is an INMS, so is $\left(A_{k}, M_{k}, \Phi_{k}\right)$.

Proof. Using (5.2), (5.3) and a result of Flanders (see [12, p. 106]), (1) is proved by induction. Using (5.5), (2) is proved by a straightforward inductive argument.

For each $a \in A$, let $\Phi^{+}(a)$ be the matrix obtained by replacing each entry of $\Phi(a)$ by its absolute value. Let $M^{+}=\sum_{a \in A} \Phi^{+}(a)$. Then we obtain an MS $\left(A, M^{+}, \Phi^{+}\right)$. It is easy to see that $\left(A^{2},\left(M^{\prime}\right)^{+},\left(\Phi^{\prime}\right)^{+}\right)=\left(A^{2},\left(M^{+}\right)^{\prime},\left(\Phi^{+}\right)^{\prime}\right)$. Hence by induction, $\left(A_{k},\left(M_{k}\right)^{+},\left(\Phi_{k}\right)^{+}\right)=\left(A_{k},\left(M^{+}\right)_{k},\left(\Phi^{+}\right)_{k}\right)$, for $k \geq 1$. Therefore since $\left(\Phi_{k}\right)^{+}(c)=0$ implies $\Phi_{k}(c)=0$ for $c \in A_{k}$, it suffices to show (3) for the case where $M$ and the $\Phi(a)$ 's are all non-negative matrices. In this case, it is clear that the $\Phi_{k}(c)$ 's, $c \in A_{k}$, and $M_{k}$ are non-negative matrices. Assume that there exists $c \in A_{k}-\tau_{k} \theta_{k}\left(A^{k}\right)$ with $\Phi_{k}(c) \neq 0$. Then $M_{k} \neq 0$ so that $M_{l} \neq 0$ for $l=1, \ldots, k$. Suppose that $u$ and $v$ have strictly positive entries. Then it is easily observed that if $M \neq 0$, then $u^{\prime}=u \Gamma$ and $v^{\prime}=\Delta v$ have strictly positive entries. Thus, by the above, $u_{k}$ and $v_{k}$ have strictly positive entries so that $u_{k} \Phi_{k}(c) v_{k} \neq 0$. This contradicts (2).

Since $\lambda u^{\prime}=\lambda u \Gamma=u M \Gamma=u \Gamma \Delta \Gamma=u^{\prime} M^{\prime}\left[\lambda v^{\prime}=\lambda \Delta v=\Delta M v=\Delta \Gamma \Delta v=M^{\prime} v^{\prime}\right]$, follows.

To see (5), let $(i, j, a),\left(i^{\prime}, j^{\prime}, b\right) \in J$ where $1 \leq i, j, i^{\prime}, j^{\prime} \leq m$ and $a, b \in A$. By definition, $\Delta((i, j, a), j)>0$ and $\Gamma\left(i^{\prime},\left(i^{\prime}, j^{\prime}, b\right)\right)>0$. Since $M$ is irreducible, there exists an integer $r>0$ such that $M^{r}\left(j, i^{\prime}\right)>0$. Therefore

$$
\left(M^{\prime}\right)^{r+1}\left((i, j, a),\left(i^{\prime}, j^{\prime}, b\right)\right)=\left(\Delta M^{r} \Gamma\right)\left((i, j, a),\left(i^{\prime}, j^{\prime}, b\right)\right)>0 .
$$

Hence $M^{\prime}$ is irreducible. Therefore, it follows by induction that $M_{k}$ is irreducible. This proves (5). 
According to (3) of the above lemma, we can define the $k$-block MS of the MS $(A, M, \Phi)$ as $\left(A^{k}, M_{k}, \Phi_{k} \tau_{k}\right)$. If $(A, M, \Phi)$ is an INMS, then so is $\left(A^{k}, M_{k}, \Phi_{k} \tau_{k}\right)$, by (5) of the above lemma.

LEMMA 5.2. Let $(A, M, \Phi)$ be an INMS and let $\bar{M}$ be a core-matrix of $(A, M, \Phi)$. Let $k$ be a positive integer. Let $\overline{M_{k}}$ be a core-matrix of the $k$-block MS $\left(A^{k}, M_{k}, \Phi_{k} \tau_{k}\right)$. Then the block of the Jordan form of $\overline{M_{k}}$ with non-zero eigenvalues is a principal submatrix of the Jordan form of $\bar{M}$.

Proof. Let $(A, \bar{M}, \bar{\Phi})$ be a core-MS of $(A, M, \Phi)$. Then there exists $(\bar{u}, \bar{v})$ such that $u_{M} \Phi(x) v_{M}=\bar{u} \bar{\Phi}(x) \bar{v}$ for all $x \in A^{*}$. Therefore, it follows from (2) of lemma 5.1 that $\left(A_{k}, M_{k}, \Phi_{k}\right)$ with $\left(\left(u_{M}\right)_{k},\left(v_{M}\right)_{k}\right)$ is equivalent to $\left(A_{k},(\bar{M})_{k},(\bar{\Phi})_{k}\right)$ with $\left((\bar{u})_{k},(\bar{v})_{k}\right)$. Let $\left(A_{k}, \bar{M}_{k}, \overline{\Phi_{k}}\right)$ be a core-MS of $\left(A_{k}, M_{k}, \Phi_{k}\right)$. Then since $\left(u_{M}\right)_{k}$ and $\left(v_{M}\right)_{k}$ are eigenvectors corresponding to the maximal characteristic value of $M_{k}$ (see (1) and (4) of lemma 5.1), there exists $(\tilde{u}, \tilde{v})$ such that $\left(A_{k}, \overline{M_{k}}, \overline{\Phi_{k}}\right)$ with $(\tilde{u}, \tilde{v})$ is reduced and equivalent to $\left(A_{k}, M_{k}, \Phi_{k}\right)$ with $\left(\left(u_{M}\right)_{k},\left(v_{M}\right)_{k}\right)$. Hence, $\left(A_{k}, \overline{M_{k}}, \overline{\Phi_{k}}\right)$ with $(\tilde{u}, \tilde{v})$ is reduced and equivalent to $\left(A_{k},(\bar{M})_{k},(\bar{\Phi})_{k}\right)$ with $\left((\tilde{u})_{k},(\bar{v})_{k}\right)$. Thus by theorem 3.1 , the Jordan form of $\overline{M_{k}}$ is a principal submatrix of that of $(\tilde{M})_{k}$ so that the result follows from (1) of lemma 5.1.

Now we are ready to prove our main theorem.

THEOREM 5.3. Let $\left(\Omega_{1}, \sigma_{1}\right)$ and $\left(\Omega_{2}, \sigma_{2}\right)$ be transitive sofic subshifts. If there exists a bounded-to-one factor map from $\left(\Omega_{1}, \sigma_{1}\right)$ to $\left(\Omega_{2}, \sigma_{2}\right)$, then the block of the Jordan form of a core-matrix $\bar{N}$ of $\left(\Omega_{2}, \sigma_{2}\right)$ with non-zero eigenvalues is a principal submatrix of the Jordan form of a core-matrix $\bar{M}$ of $\left(\Omega_{1}, \sigma_{1}\right)$.

Proof. Let $(A, M, \Phi)$ and $(B, N, \Psi)$ be normal INMS's such that $L\left(\Omega_{1}\right)=L_{(A, M, \Phi)}$ and $L\left(\Omega_{2}\right)=L_{(B, N, \Psi)}$ and $\bar{M}$ and $\bar{N}$ are core-matrices of $(A, M, \Phi)$ and $(B, N, \Psi)$, respectively. Let $\pi:\left(\Omega_{1}, \sigma_{1}\right) \rightarrow\left(\Omega_{2}, \sigma_{2}\right)$ be a bounded-to-one and onto $k$-block factor map with $k \geq 1$. Define a $k$-block map $\phi: \Omega_{1} \rightarrow\left(A^{k}\right)^{\mathbb{Z}}$ by $\phi(\alpha)=\gamma$ where $\gamma_{i}=$ $\alpha_{i} \cdots \alpha_{i+k-1}, \alpha \in \Omega_{1}, i \in \mathbb{Z}$. Let $\phi\left(\Omega_{1}\right)=\Omega$. We obtain a subshift $(\Omega, \sigma)$, which is well known in terms of a higher block system [1]. Clearly, $\phi$ is injective and there exists a 1-block map $\tilde{\pi}: \Omega \rightarrow \Omega_{2}$ such that $\pi=\tilde{\pi} \phi$. It is also clear that $\theta_{k}\left(A^{k} A^{*} \cap L\left(\Omega_{1}\right)\right)=L(\Omega)$. It follows from (2) of lemma 5.1 that $L(\Omega)=L_{\left(A^{k}, M_{k}, \Phi_{k} \tau_{k}\right)}$. Since $(A, M, \Phi)$ is a normal INMS, so is $\left(A^{k}, M_{k}, \Phi_{k} \tau_{k}\right)$ by (2) and (5) of lemma 5.1. Therefore, since $\tilde{\pi}$ is bounded-to-one and onto, it follows from theorem 4.1 that the Jordan form of $\bar{N}$ is a principal submatrix of the Jordan form of a core-matrix $\overline{M_{k}}$ of $\left(A^{k}, M_{k}, \Phi_{k} \tau_{k}\right)$. Thus by lemma 5.2, the block of the Jordan form of $\bar{N}$ with non-zero eigenvalues is a principal submatrix of the Jordan form of $\bar{M}$.

The above theorem is an extension of a result of Kitchens [14, corollary A].

Example. Let $A=\{a, b, c\}$. Let $(A, M, \Phi)$ be the INMS with

$$
\Phi(a)=\left(\begin{array}{lll}
0 & 1 & 0 \\
1 & 0 & 1 \\
0 & 0 & 0
\end{array}\right), \quad \Phi(b)=\left(\begin{array}{lll}
0 & 0 & 1 \\
0 & 0 & 0 \\
0 & 1 & 0
\end{array}\right), \quad \Phi(c)=\left(\begin{array}{lll}
0 & 0 & 0 \\
0 & 0 & 0 \\
1 & 0 & 0
\end{array}\right)
$$


and let $(A, N, \Psi)$ be the INMS with

$$
\Psi(a)=\left(\begin{array}{lll}
0 & 1 & 0 \\
1 & 0 & 1 \\
0 & 0 & 0
\end{array}\right), \quad \Psi(b)=\left(\begin{array}{lll}
0 & 0 & 1 \\
0 & 0 & 0 \\
0 & 1 & 0
\end{array}\right), \quad \Psi(c)=\left(\begin{array}{lll}
0 & 0 & 0 \\
0 & 0 & 0 \\
0 & 1 & 0
\end{array}\right) .
$$

It is easy to see that both of $(A, M, \Phi)$ and $(A, N, \Psi)$ are normal and core-MS's. The elementary divisors of $M$ are $\lambda-2, \lambda+1$, and $\lambda+1$ but the elementary divisors of $N$ are $\lambda-2$ and $(\lambda+1)^{2}$. Hence by theorem 5.3 , there exists no bounded-to-one factor map between the transitive sofic subshifts $\left(\Omega_{1}, \sigma_{1}\right)$ with $L\left(\Omega_{1}\right)=L_{(A, M, \Phi)}$ and $\left(\Omega_{2}, \sigma_{2}\right)$ with $L\left(\Omega_{2}\right)=L_{(A, N, \Psi)}$.

\section{Sofic subshifts that are spectrally of finite type}

A transitive sofic subshift $(\Omega, \sigma)$ is spectrally of finite type (SFT) if there exist a transitive subshift of finite type $\left(\Omega_{0}, \sigma_{0}\right)$, a sofic subshift $\left(\Omega_{1}, \sigma_{1}\right)$ which is topologically conjugate to $(\Omega, \sigma)$ and has a core-matrix similar to that of $\left(\Omega_{0}, \sigma_{0}\right)$, and a bounded-to-one 1-block factor map from $\left(\Omega_{0}, \sigma_{0}\right)$ to $\left(\Omega_{1}, \sigma_{1}\right)$. We conjecture that all transitive sofic subshifts are SFT and hence transitive sofic subshifts inherit their spectral structure completely from subshifts of finite type. In fact, no example is known even for a transitive sofic subshift which has no $\lambda$-graph as its core-MS. If an irreducible $\lambda$-graph $(A, M, \Phi)$ is a core-MS of a transitive sofic subshift $(\Omega, \sigma)$, then $(A, M, \Phi)$ describes a bounded-to-one 1-block factor map from the irreducible subshift of finite type $\left(\Omega_{M}, \sigma_{M}\right)$ described by $M$ to $(\Omega, \sigma)$ and $M$ is a core-matrix of both of $(\Omega, \sigma)$ and $\left(\Omega_{M}, \sigma_{M}\right)$. It has not been proved, however, that the conjecture is true. Here, we only prove that the subshifts that are 'almost of finite type' in the sense of Marcus [16], are SFT.

A factor map is right [left] closing if it never collapses two left [right] asymptotic points [13]. A sofic subshift is almost of finite type (AFT) if it is the factor of an irreducible subshift of finite type by a both right and left closing factor map. For the original definition of AFT, see [16].

A $\lambda$-graph is right [left] closing (or bundle-mergible [backward bundle-mergible] [19]) if there exists an integer $k \geq 0$ such that for any $l \geq k$ and for any two paths of length $l$ generating the same word and having the same initial [terminal] vertex, the initial [terminal] subpaths of length $l-k$ of them coincide. It is easy to see that a sofic subshift is AFT iff there exists a both right and left closing (or mergible [19]) irreducible $\lambda$-graph $(A, M, \Phi)$ such that $L(\Omega)=L_{(A, M, \Phi)}$.

Boyle [2] has shown an example of a transitive sofic subshift which is not AFT. Moreover the following theorem gives a simple criterion to determine whether a given sofic subshift is AFT.

THEOREM 6.1. Let $(\Omega, \sigma)$ be a transitive sofic subshift and let $(A, M, \Phi)$ be the minimal right resolving $\lambda$-graph such that $L_{(A, M, \Phi)}=L(\Omega)$. Then $(\Omega, \sigma)$ is AFT iff $(A, M, \Phi)$ is left-closing.

Proof. In [19], the 'induced regular homomorphism' was constructed for any homomorphism between strongly connected graphs whose global map is finite-to-one 
and onto. The same construction gives the induced right resolving $\lambda$-graph for any normal $\lambda$-graph if we take the maximal compatible sets and all of their successors as the vertex (point) set of the $\lambda$-graph to be constructed (see $[19, \S 4]$ ). It is easily seen that this construction preserves irreducibility (of $\lambda$-graphs). Of course, a $\lambda$-graph and its induced right resolving $\lambda$-graph generate the same language. By the proof of lemma 5.4 of [19], it is easy to see that the corresponding result of the lemma for $\lambda$-graphs also holds. That is, if a $\lambda$-graph is both right and left closing, then its induced right resolving $\lambda$-graph is left closing.

If $(\Omega, \sigma)$ is AFT, then there exists a both right and left closing irreducible $\lambda$-graph $\left(A, M^{\prime}, \Phi^{\prime}\right)$ such that $L(\Omega)=L_{\left(A, M^{\prime}, \Phi^{\prime}\right)}$. By the above, the induced right resolving $\lambda$-graph $\left(A, M^{\prime \prime}, \Phi^{\prime \prime}\right)$ of $\left(A, M^{\prime}, \Phi^{\prime}\right)$ is irreducible and left closing and $L(\Omega)=$ $L_{\left(A, M^{\prime \prime}, \Phi^{\prime \prime}\right)}$. If we minimize $\left(A, M^{\prime \prime}, \Phi^{\prime \prime}\right)$ in the sense of state minimization in finite automata theory (see e.g. [9]), we obtain $(A, M, \Phi)$, by lemma 3 of [7].

Two vertices (states) $s_{1}$ and $s_{2}$ of the right resolving $\lambda$-graph $\Gamma=\left(A, M^{\prime \prime}, \Phi^{\prime \prime}\right)$ are equivalent if for each $x \in A^{*}$, there exists a path going from $s_{1}$ and generating $x$ when and only when there exists a path going from $s_{2}$ and generating $x$. Then $(A, M, \Phi)$ is the $\lambda$-graph $\tilde{\Gamma}$ defined as follows. The vertices of $\tilde{\Gamma}$ are the equivalence classes of vertices of $\Gamma$. For each pair of equivalence classes $C_{1}$ and $C_{2}$ and each $a \in A$, a (unique) arc labelled by $a$ goes from $C_{1}$ to $C_{2}$ in $\tilde{\Gamma}$ if there exists an arc of $\Gamma$ which is labelled by $a$ and goes from a vertex in $C_{1}$ to a vertex in $C_{2}$. Let $\mathscr{A}$ be the arc set of $\Gamma$ and $\tilde{A}$ that of $\tilde{\Gamma}$. We define $h: \mathscr{A} \rightarrow \tilde{A}$ as follows: if $z \in \mathscr{A}$ goes from a vertex in an equivalence class $C_{1}$ to a vertex in an equivalence class $C_{2}$ and is labelled by $a \in A$, then $h(z)$ is the arc labelled by $a$ going from $C_{1}$ to $C_{2}$ in $\tilde{\Gamma}$. We define a 1-block factor map $\pi_{1}$ between the irreducible subshifts of finite type $\left(\Omega_{M^{\prime \prime}}, \sigma_{M^{\prime \prime}}\right)$ described by $M^{\prime \prime}$ and $\left(\Omega_{M}, \sigma_{M}\right)$ described by $M$ as follows: for each $\alpha \in \Omega_{M^{\prime \prime}},\left(\pi_{1}(\alpha)\right)_{i}=h\left(\alpha_{i}\right)$ for all $i \in \mathbb{Z}$. Let $\pi_{2}$ be the 1-block factor map from $\left(\Omega_{M}, \sigma_{M}\right)$ to $(\Omega, \sigma)$ described by $(A, M, \Phi)$. Let $\pi=\pi_{2} \pi_{1}$. Then clearly $\pi$ is the 1-block factor map from $\left(\Omega_{M^{\prime \prime}}, \sigma_{M^{\prime \prime}}\right)$ to $(\Omega, \sigma)$ described by $\left(A, M^{\prime \prime}, \Phi^{\prime \prime}\right)$. Since $\pi$ is both right and left closing, it follows that so is $\pi_{1}$. Thus by theorem 6.3 of [19], $\pi_{1}$ is constant-to-one. Assume that $\pi_{2}$ is not left-closing. Then there exist $\beta, \beta^{\prime} \in \Omega_{M}$ such that $\beta \neq \beta^{\prime}, \beta_{i}=\beta_{i}^{\prime}$ for all $i \geq 0$, and $\pi_{2}(\beta)=\pi_{2}\left(\beta^{\prime}\right)$. If $\alpha \in \pi_{1}^{-1}(\beta)$ and $\alpha^{\prime} \epsilon$ $\pi_{1}^{-1}\left(\beta^{\prime}\right)$, then $\alpha$ and $\alpha^{\prime}$ are not right asymptotic because otherwise, $\pi$ would not be left closing. Hence

$$
\left|\pi_{1}^{-1}(\gamma)\right| \geq\left|\pi_{1}^{-1}(\beta)\right|+\left|\pi_{1}^{-1}\left(\beta^{\prime}\right)\right|
$$

for a point $\gamma$ in the $\omega$-limiting set of $\beta$, which is a contradiction because $\pi_{1}$ is constant-to-one. Thus $(A, M, \Phi)$ is left closing.

The converse is clear.

Remark 6.2. The following also gives a simple criterion to determine whether a given transitive sofic subshift is a subshift of finite type. Let $(\Omega, \sigma)$ be a transitive sofic subshift and let $(A, M, \Phi)$ be the minimal right resolving $\lambda$-graph such that $L_{(A, M, \Phi)}=L(\Omega)$. Then $(\Omega, \sigma)$ is of finite type iff $(A, M, \Phi)$ is definite (see [19]), i.e. there exists an integer $k \geq 0$ such that $\mathbb{\Phi} \Phi(x)$ has at most one non-zero entry for all $x \in A^{k}$, where $\mathbb{1}$ is the row vector with all entries 1 . 
Remark 6.3. From the observation by Boyle [2, § III] on the work of Kitchens [13] or by straightforward modifications of the arguments of $[19, \S 5]$, we have a result analogous to theorem 5.9 of [19]. That is, for any both right and left closing factor map $\phi$ from an irreducible subshift of finite type $\left(\Omega_{1}, \sigma_{1}\right)$ to a transitive sofic subshift $\left(\Omega_{2}, \sigma_{2}\right)$, there exists a both right and left resolving irreducible $\lambda$-graph $(B, N, \Psi)$ with $L_{(B, N, \Psi)}=L\left(\hat{\Omega}_{2}\right)$ for some higher block system $\left(\hat{\Omega}_{2}, \hat{\sigma}_{2}\right)$ of $\left(\Omega_{2}, \sigma_{2}\right)$, an isomorphism $\rho_{1}:\left(\Omega_{1}, \sigma_{1}\right) \rightarrow\left(\Omega_{N}, \sigma_{N}\right)$, and a natural isomorphism $\rho_{2}:\left(\hat{\Omega}_{2}, \hat{\sigma}_{2}\right) \rightarrow\left(\Omega_{2}, \sigma_{2}\right)$ such that $\phi=\rho_{2} \psi \rho_{1}$, where $\left(\Omega_{N}, \sigma_{N}\right)$ is the irreducible subshift of finite type described by $N$ and $\psi:\left(\Omega_{N}, \sigma_{N}\right) \rightarrow\left(\hat{\Omega}_{2}, \hat{\sigma}_{2}\right)$ is the 1 -block factor map described by $(B, N, \Psi)$.

THEOREM 6.4. An AFT sofic subshift is SFT.

Proof. Let $(\Omega, \sigma)$ be an AFT sofic subshift. Let $(A, M, \Phi)$ be the minimal right resolving $\lambda$-graph such that $L(\Omega)=L_{(A, M, \Phi)}$. Let $\phi$ be the 1-block factor map described by $(A, M, \Phi)$. By theorem $6.1, \phi$ is both right and left closing. Since $(A, M, \Phi)$ is the minimal right resolving $\lambda$-graph, it has a magic word, by the proof of lemma 2 of [6]. Therefore $\left|\phi^{-1}(\alpha)\right|=1$ for a bilaterally transitive point $\alpha \in \Omega$ (see $[1$, p. 25]). Applying the fact stated in remark 6.3 to $\phi$, we have a both right and left resolving $\lambda$-graph $(B, N, \Psi)$ such that $L_{(B, N, \Psi)}=L(\hat{\Omega})$ for some higher block system $(\hat{\Omega}, \hat{\sigma})$ of $(\Omega, \sigma)$ and the 1 -block factor map $\psi$ described by $(B, N, \Psi)$ has the property that $\left|\psi^{-1}(\beta)\right|=1$ for a bilaterally transitive point $\beta \in \hat{\Omega}$. Thus the both right and left resolving $\lambda$-graph $(B, N, \Psi)$ has a magic word both to the right and to the left (see [1, p. 26]). Therefore it follows from the proof of proposition 3.3 that $(B, N, \Psi)$ is a core-MS of $(\hat{\Omega}, \hat{\sigma})$. Thus $(\Omega, \sigma)$ is SFT.

We remark that the proof of theorem 6.4 also shows that the definition of AFT stated above is equivalent to the original one given by Marcus [16].

\section{Concluding Remarks}

Let $(A, M, \Phi)$ be an NMS of order $m$ with a pair $(u, v)$ of non-negative row and column vectors such that $u M=u, M v=v, u \nabla_{m}^{t}=1$, and $u v=1$. Let $(\Omega, \sigma)$ be a sofic subshift such that $L(\Omega) \supset\left\{x \in A A^{*} \mid u \Phi(x) v>0\right\}$. Then we can equip $(\Omega, \sigma)$ with the invariant Borel probability measure $\mu$ defined by $(A, M, \Phi)$ with $(u, v)$. That is, for each cylinder set

we define

$$
{ }_{i}[x]_{j}=\left\{\alpha \in \Omega \mid \alpha_{i} \alpha_{i+1} \cdots \alpha_{j}=x\right\}, \quad x \in A^{j-i+1} \cap L(\Omega),
$$

$$
\mu\left({ }_{i}[x]_{j}\right)=u \Phi(x) v .
$$

Let $\left(\Omega_{i}, \sigma_{i}\right)$ be a sofic subshift equipped with the measure $\mu_{i}$ defined by $\left(A_{i}, M_{i}, \Phi_{i}\right)$ with $\left(u_{i}, v_{i}\right)$ as above, for $i=1,2$. Let $\left(A_{i}, \bar{M}_{i}, \bar{\Phi}_{i}\right)$ with $\left(\bar{u}_{i}, \bar{v}_{i}\right)$ be the reduced form of $\left(A_{i}, M_{i}, \Phi_{i}\right)$ with $\left(u_{i}, v_{i}\right)$, for $i=1,2$. Then one can show that if there exists a measure preserving factor map from $\left(\Omega_{1}, \sigma_{1}\right)$ to $\left(\Omega_{2}, \sigma_{2}\right)$, then the block of the Jordan form of $\bar{M}_{2}$ with non-zero eigenvalues is a principal submatrix of the Jordan form of $\bar{M}_{1}$. This is a generalization of the theorem of Kitchens in [14].

Let $(A, M, \Phi)$ be an INMS. Let $(\Omega, \sigma)$ be the sofic subshift such that $L(\Omega)=$ $L_{(A, M, \Phi)}$. Then we can equip $(\Omega, \sigma)$ with the measure $\mu$ defined by $(A, \hat{M}, \hat{\Phi})$ with $\left(u_{M}, v_{M}\right)$ as above, where $\hat{M}=\left(1 / \lambda_{M}\right) M$ and $\hat{\Phi}(a)=\left(1 / \lambda_{M}\right) \Phi(a)$ for each $a \in A$. 
By lemma 2.2, $\mu$ is ergodic so that the proof of theorem 2.3 can be reduced to a property of ergodic invariant Borel probability measures (see [22, p. 153]). We also remark that $\log \lambda_{M}$ is the topological entropy and $\mu$ is a (unique) measure of maximal entropy iff $(A, M, \Phi)$ is normal. Therefore, one can also use an approach parallel to that of [14] to prove theorem 5.3.

At the MSRI workshop on Coding and Isomorphism in Ergodic Theory held in December 1983, I learned that in [15] W. Krieger proved the following: if two transitive sofic subshifts $\left(\Omega_{1}, \sigma_{1}\right)$ and $\left(\Omega_{2}, \sigma_{2}\right)$ are topologically conjugate and $\left(A_{i}, M_{i}, \Phi_{i}\right)$ is the minimal right [left] resolving $\lambda$-graph for $\left(\Omega_{i}, \sigma_{i}\right)$ (i.e. $L_{\left(A_{i}, M_{i} \Phi_{i}\right)}=$ $\left.L\left(\Omega_{i}\right)\right)$ for $i=1,2$, then $\left(\Omega_{M_{1}}, \sigma_{M_{1}}\right)$ and $\left(\Omega_{M_{2}}, \sigma_{M_{2}}\right)$ are topologically conjugate, where $\left(\Omega_{M_{i}}, \sigma_{M_{i}}\right)$ is the subshift of finite type described by $M_{i}$ for $i=1,2$. Let $(A, \vec{M}, \vec{\Phi})$ and $(A, \bar{M}, \overleftarrow{\Phi})$ be the minimal right and left resolving $\lambda$-graphs for a transitive sofic subshift $(\Omega, \sigma)$ and let $\bar{M}$ be a core-matrix of $(\Omega, \sigma)$. Then by Krieger's result, the blocks of the Jordan forms of $\vec{M}$ and $\dot{M}$ with non-zero eigenvalues are invariants for topological conjugacy for transitive sofic subshifts. As stated in our main theorem, the block of the Jordan form of $\bar{M}$ with non-zero eigenvalues is an invariant for bounded-to-one factor maps (and hence for topological conjugacy). It follows from the proof of theorem 6.4, however, that all of these three invariants are the same when $(\Omega, \sigma)$ is AFT. But the following example shows that they are different in general and neither of the blocks of the Jordan forms of $\vec{M}$ and $\bar{M}$ with non-zero eigenvalues is an invariant for bounded-to-one factor maps.

Example. Let $(A, M, \Phi)$ be the INMS ( $\lambda$-graph) given by $A=\{a, b, c\}$ and

or

$$
\Phi(a)=\left(\begin{array}{lll}
1 & 0 & 0 \\
0 & 0 & 1 \\
0 & 1 & 0
\end{array}\right), \quad \Phi(b)=\left(\begin{array}{lll}
0 & 1 & 1 \\
0 & 1 & 0 \\
0 & 0 & 0
\end{array}\right), \quad \Phi(c)=\left(\begin{array}{lll}
0 & 0 & 0 \\
0 & 0 & 0 \\
1 & 0 & 0
\end{array}\right) \text {, }
$$

$$
a \Phi(a)+b \Phi(b)+c \Phi(c)=\left(\begin{array}{lll}
a & b & b \\
0 & b & a \\
c & a & 0
\end{array}\right)
$$

Then it is easily seen that $(A, M, \Phi)$ is normal and is a core-MS. Hence $M$ is a core-matrix $\bar{M}$ of the sofic subshift $(\Omega, \sigma)$ with $L(\Omega)=L_{(A, M, \Phi)}$, and the characteristic polynomial of $\bar{M}$ is $\lambda^{3}-2 \lambda^{2}-\lambda+1$. The minimal right and left resolving $\lambda$-graphs $(A, \vec{M}, \vec{\Phi})$ and $(A, \hat{M}, \overleftarrow{\Phi})$ for $(\Omega, \sigma)$ are given by

and

$$
a \vec{\Phi}(a)+b \vec{\Phi}(b)+c \vec{\Phi}(c)=\left(\begin{array}{cccc}
a & 0 & 0 & b \\
0 & b & a & 0 \\
c & a & 0 & 0 \\
c & b & 0 & a
\end{array}\right)
$$

$$
a \overleftarrow{\Phi}(a)+b \overleftarrow{\Phi}(b)+c \overleftarrow{\Phi}(c)=\left(\begin{array}{ccccc}
a & 0 & b & 0 & b \\
0 & 0 & a & 0 & 0 \\
c & a & 0 & c & c \\
0 & b & 0 & b & a \\
0 & 0 & 0 & a & 0
\end{array}\right) .
$$


The characteristic polynomials of $\vec{M}$ and $\bar{M}$ are $(\lambda-1)\left(\lambda^{3}-2 \lambda^{2}-\lambda+1\right)$ and $(\lambda-1)(\lambda+1)\left(\lambda^{3}-2 \lambda^{2}-\lambda+1\right)$, respectively.

I would like to thank Brian Marcus and Mike Boyle for stimulating discussions on core-matrices at the MSRI workshop. Marcus's questions prompted the addition of the above remark. I also learned that theorem 6.1 was also proved in [3] together with more about AFT sofic subshifts.

\section{REFERENCES}

[1] R. L. Adler \& B. Marcus. Topological entropy and equivalence of dynamical systems. Mem. Amer. Math. Soc. 219 (1979).

[2] M. Boyle. Topological orbit equivalence and factor maps in symbolic dynamics. Ph. D. Thesis, University of Washington, Seattle (1983).

[3] M. Boyle, B. Kitchens \& B. Marcus. A note on minimal covers for sofic systems. Preprint.

[4] E. M. Coven \& M. E. Paul. Sofic systems, Israel J. Math. 20 (1975), 165-177.

[5] E. M. Coven \& M. E. Paul. Finite procedures for sofic systems. Monatsh. Math. 83 (1977), 265-278.

[6] R. Fischer. Sofic systems and graphs, Monatsh. Math. 80 (1975), 179-186.

[7] R. Fischer. Graphs and symbolic dynamics. In Colloq. Math. Soc. János Bolyai 16, Topics in Information Theory. Keszthely: Hungary, 1975.

[8] F. R. Gantmacher. The Theory of Matrices, Vol. II. Chelsea: New York, 1959.

[9] M. A. Harrison. Introduction to Switching and Automata Theory. McGraw-Hill: New York, 1965.

[10] G. A. Hedlund. Endomorphisms and automorphisms of the shift dynamical system. Math. Syst. Theory 3 (1969), 320-375.

[11] Y. Inagaki, T. Fukumura \& H. Matuura. Some aspects of linear space automata. Inform. and Contr. 20 (1972), 439-479.

[12] N. Jacobson. Lectures in Abstract Algebra, Vol. II, Springer-Verlag: New York, 1953.

[13] B. Kitchens. Continuity properties of factor maps in ergodic theory. $\mathrm{Ph}$. D. Thesis, University of North Carolina, Chapel Hill (1981).

[14] B. Kitchens. An invariant for continuous factors of Markov shifts. Proc. Amer. Math. Soc. 83 (1981), 825-828.

[15] W. Krieger. On sofic systems I. Preprint, Institut für Angewandte Mathematik der Universität Heidelberg.

[16] B. Marcus. Sofic systems and encoding data. Preprint, Department of Mathematics, University of North Carolina.

[17] H. Matuura, Y. Inagaki \& T. Fukumura. A generalization of automata and its analysis. Records Natl. Convention, IECE, Japan A67-45 (1968). (In Japanese.)

[18] M. Nasu. Uniformly finite-to-one and onto extensions of homomorphisms between strongly connected graphs. Discrete Math. 39 (1982), 171-197.

[19] M. Nasu. Constant-to-one and onto global maps of homomorphisms between strongly connected graphs. Ergod. Th. \& Dynam. Sys. 3 (1983), 387-413.

[20] W. Parry. A finitary classification of topological Markov chains and sofic systems. Bull. London Math. Soc. 9 (1977), 86-92.

[21] A. Paz. Introduction to Probabilistic Automata. Academic Press: New York, 1971.

[22] P. Walters. An Introduction to Ergodic Theory. Springer-Verlag: New York, 1982.

[23] B. Weiss. Subshifts of finite type and sofic systems. Monatsh. Math. 77 (1973), 462-474. 